\section{6 OPEN ACCESS}

\title{
Anti-IP-10 antibody (BMS-936557) for ulcerative colitis: a phase II randomised study
}

\author{
Lloyd Mayer, ${ }^{1}$ William J Sandborn, ${ }^{2}$ Yuriy Stepanov, ${ }_{1}^{3}$ Karel Geboes, ${ }_{1}^{4}$ Robert Hardi, ${ }^{5}$ \\ Michael Yellin, ${ }^{6}$ Xiaolu Tao, ${ }^{7}$ Li An Xu, ${ }^{7}$ Luisa Salter-Cid, ${ }^{7}$ Sheila Gujrathi, ${ }^{7}$ \\ Richard Aranda, ${ }^{7}$ Allison Y Luo ${ }^{7}$
}

\begin{abstract}
- Additional material is published online only. To view please visit the journal online (http://dx.doi.org/10.1136/ gutjnl-2012-303424).

${ }^{1}$ Immunology Institute, Mount Sinai School of Medicine, New York, New York, USA ${ }^{2}$ Division of Gastroenterology, University of California San Diego, La Jolla, California, USA ${ }^{3}$ Dnipropetrovsk State Medical Academy, Dnipropetrovsk, Ukraine

${ }^{4}$ Department of Pathologie, University Hospital KU Leuven, Leuven, Belgium

${ }^{5}$ Chevy Chase Clinical

Research, Chevy Chase, Maryland, USA

${ }^{6}$ Medarex Inc, Bloomsbury, New Jersey, USA

${ }^{7}$ Bristol-Myers Squibb,

Princeton, New Jersey, USA
\end{abstract}

\section{Correspondence to}

Dr Lloyd Mayer,

Immunobiology and

Microbiology, Immunology

Institute, Mount Sinai School

of Medicine, 1425 Madison

Avenue, 11th Floor, New York, NY 10029, USA;

Lloyd.mayer@mssm.edu

Received 26 July 2012 Revised 10 January 2013 Accepted 11 January 2013 Published Online First

5 March 2013

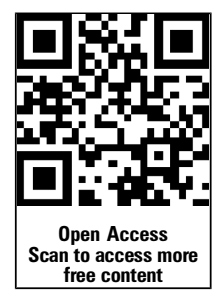

To cite: Mayer $L$, Sandborn WJ, Stepanov Y, et al. Gut 2014:63: 442-450.

\section{ABSTRACT}

Objective Interferon- $\gamma$-inducible protein-10 (IP-10 or CXCL10) plays a role in inflammatory cell migration and epithelial cell survival and migration. It is expressed in higher levels in the colonic tissue and plasma of patients with ulcerative colitis (UC). This phase II study assessed the efficacy and safety of BMS-936557, a fully human, monoclonal antibody to IP-10, in the treatment of moderately-to-severely active UC.

Design In this 8-week, phase II, double-blind, multicentre, randomised study, patients with active UC received placebo or BMS-936557 (10 mg/kg) intravenously every other week. The primary endpoint was the rate of clinical response at Day 57; clinical remission and mucosal healing rates were secondary endpoints. Post hoc analyses evaluated the drug exposure-response relationship and histological improvement.

Results 109 patients were included (BMS-936557: $n=55$; placebo: $n=54$ ). Prespecified primary and secondary endpoints were not met; clinical response rate at Day 57 was $52.7 \%$ versus $35.2 \%$ for BMS-936557 versus placebo $(p=0.083)$, and clinical remission and mucosal healing rates were $18.2 \%$ versus $16.7 \%$ $(p=1.00)$ and $41.8 \%$ versus $35.2 \%(p=0.556)$, respectively. However, higher BMS-936557 steady-state trough concentration $\left(C_{\text {minss }}\right)$ was associated with increased clinical response $(87.5 \%$ vs $37.0 \%(p<0.001)$ for patients with $C_{\text {minss }} 108-235 \mu \mathrm{g} / \mathrm{ml}$ vs placebo) and histological improvements $(73.0 \%$ vs $41.0 \% ; \mathrm{p}=0.004)$. Infections occurred in 7 (12.7\%) BMS-936557-treated patients and $3(5.8 \%)$ placebo-treated patients. 2 (3.6\%) BMS-936557 patients discontinued due to adverse events.

Conclusions Anti-IP-10 antibody, BMS-936557, is a potentially effective therapy for moderately-to-severely active UC. Higher drug exposure correlated with increasing clinical response and histological improvement. Further dose-response studies are warranted.

Clinical Trial Registration Number: ClinicalTrials. gov NCT00656890.

\section{INTRODUCTION}

Ulcerative colitis (UC) is a chronic disease characterised by relapsing-remitting inflammation of the colonic mucosa, believed to be multifactorial in disease pathophysiology, including an aberrant

\section{Significance of this study}

What is already known on this subject?

- Ulcerative colitis (UC) is a chronic, inflammatory disease of the colonic mucosa caused, in part, by an aberrant immune system.

- Current treatments achieve remission in a relatively small proportion of patients and can be associated with toxicities, such as infections and malignancies.

- Interferon- $\gamma$-inducible protein-10 (IP-10) is an endogenous chemokine that is directly, and indirectly, involved in inflammatory cell migration and epithelial cell survival.

- IP-10 is expressed at higher levels in the colonic tissue and plasma of patients with UC and may contribute to the chronic intestinal inflammation and tissue damage observed in such patients.

What are the new findings?

- Inhibiting IP-10 activity through the use of the monoclonal antibody, BMS-936557, demonstrated efficacy in patients who achieved high serum concentrations in this phase II, randomised, controlled, proof-of-concept study of moderate-to-severe UC.

- Higher drug exposure resulted in increased rates of clinical response and clinical remission, in addition to histological improvements, versus placebo.

- Treatment was generally well tolerated in this patient population with relatively low rates of adverse events, including infections and infusional events; no deaths, malignancies or positive immunogenicity were reported.

How might it impact on clinical practice in the foreseeable future?

- These preliminary data demonstrate that therapies that inhibit IP-10-mediated inflammatory response and modulate epithelial homeostasis may provide an effective and well-tolerated approach for the treatment of UC. Further dose-response studies are required to establish optimal efficacy with an acceptable risk-benefit profile. 
immune response. ${ }^{1}$ Conventional treatment for UC involves the use of aminosalicylates (ASA), corticosteroids, immunomodulators (azathioprine (AZA), 6-mercaptopurine (6-MP) and cyclosporine) and biological therapy targeting specific cytokines, such as the antitumour necrosis factor (anti-TNF) antibody, infliximab. ${ }^{2}{ }^{3}$ However, not all patients will achieve remission, and corticosteroids, immunomodulators and anti-TNF therapy can be associated with toxicities, including infection and malignancy, rendering treatment inappropriate for some patients. ${ }^{4}$ As such, there is a need for new therapies with alternative mechanisms of action that offer a more favourable risk-benefit profile.

Interferon- $\gamma$-inducible protein-10 (IP-10), also referred to as CXCL10, is a chemokine that plays an important role in the integrin activation and migration of cells, including activated $\mathrm{T}$ cells, monocytes, eosinophils, natural killer, epithelial and endothelial cells. ${ }^{5}$ The receptor for IP-10 is chemokine (cys- $x$-cys motif) receptor 3 (CXCR3). Stimulation by IP-10 is directly and indirectly involved in the generation and recruitment of the pro-inflammatory cells that are responsible for inflammation and tissue destruction. ${ }^{6} 7$ IP-10 exerts its chemotactic activity on T helper (Th) 1 and Th17 cells, and blockade of IP-10 may skew cytokine activity from a Th1 to a Th2 profile. $^{8}$ Thus, this chemokine can regulate inflammation by both induction of cellular migration and promotion of a Th1/ Th17 response. IP-10 also appears to modulate cellular functions independently of CXCR3, including epithelial cell proliferation and migration as well as endothelial cell proliferation via heparan sulfate. ${ }^{9-12}$ These additional mechanisms of action may also contribute to the potential role of IP-10 in driving intestinal inflammation.

Expression of IP-10 in colonic tissue and IP-10 plasma concentrations are greater in patients with UC compared with healthy controls. ${ }^{13} 14$ In experimental murine models, treatment with anti-IP-10 antibodies protected against epithelial ulceration in a dextran sodium sulfate injury/repair model, ${ }^{15}$ reduced inflammation in IL- $10^{-/-}$mice, ${ }^{16}$ and reduced colitis by impairing Th1 induction and recruitment. ${ }^{17}$ These findings suggest that anti-IP-10 therapy may be a novel treatment option for UC.

BMS-936557 (previously termed MDX-1100) is a fully human monoclonal antibody that targets IP-10. BMS-936557 binds to IP-10 with high affinity $(\sim 1 \mathrm{nM})$ and specifically blocks its interaction with CXCR3. ${ }^{18}$ As a result, BMS-936557 is a potent inhibitor of IP-10-induced chemotaxis of activated T cells $\left(\mathrm{EC}_{50} \sim 0.8 \mathrm{nM}\right) .{ }^{18}$ BMS-936557 does not bind to either Mig (CXCL9) or ITAC (CXCL11), the other ligands for CXCR3, ${ }^{18}$ and so is only expected to modulate IP-10-mediated functions. Phase I studies have evaluated the pharmacodynamic, pharmacokinetic and safety profile of BMS-936557 at doses of up to $10 \mathrm{mg} / \mathrm{kg}$ in healthy volunteers, in addition to demonstrating safety in patients with UC. ${ }^{19}{ }^{20}$ A phase II study subsequently demonstrated safety and potential efficacy of BMS-936557 $(10 \mathrm{mg} / \mathrm{kg})$ in patients with rheumatoid arthritis refractory to methotrexate therapy. ${ }^{20}$

We conducted a phase II, 8-week induction study of BMS-936557 in patients with moderately-to-severely active UC.

\section{METHODS}

\section{Study design and patient population}

This was an 8-week, phase II, double-blind, placebo-controlled, randomised, multicentre study of BMS-936557 (trial identifier: NCT00656890; URL: http://clinicaltrials.gov/ct2/show/NCT00 656890), conducted at 54 medical centres in eight countries (Ukraine, Hungary, Latvia, USA, Czech Republic, Romania, Canada and Russia) between 26 March 2008 and 14 September
2009. All patients gave written, informed consent and the study was approved by local ethics committees and conducted in accordance with Good Clinical Practice guidelines and the Declaration of Helsinki.

Eligible patients were those $\geq 18$ years of age with an active UC disease flare while on stable doses of 5-ASA, corticosteroids, AZA and/or 6-MP. Disease flare was defined as a Mayo score of 6-10 with a Mayo endoscopic subscore $\geq 2$ within 2 weeks before study drug administration. ${ }^{21} 22$ Excluded patients included those with Cushing's disease, primary or secondary immunodeficiency, toxic megacolon or fulminant disease likely to require colectomy, acute or chronic infection, including Clostridium difficile, active tuberculosis, or Herpes zoster $\leq 3$ months prior to study drug administration. The use of anti-TNF therapy or any monoclonal antibody or immunoglobulin-based fusion protein within 8 weeks prior to study treatment, or any experimental therapy within 4 weeks prior to study drug administration, was prohibited.

\section{Randomisation, treatment administration and dosage}

Patients were randomised in a 1:1 ratio to receive intravenous BMS-936557 $(10 \mathrm{mg} / \mathrm{kg})$, or placebo, at Weeks $0,2,4$ and 6 (maximum dose $1000 \mathrm{mg}$ ). After this treatment period, patients were followed through Day 113. Randomisation was performed centrally using dynamic treatment allocation. Treatment assignment was blinded for personnel at the study sites and for patients; the study site pharmacist/designated nurse was unblinded for study drug preparation. Blinding was maintained throughout the study. Oral 5-ASA, prednisolone $\leq 20 \mathrm{mg} /$ day, AZA and 6-MP were continued at stable doses during the study.

\section{Efficacy assessments}

Prespecified analyses

The primary efficacy endpoint was the rate of clinical response at Day 57, defined as a decrease from baseline in the Mayo total score $\geq 3$ points and $\geq 30 \%$, with an accompanying decrease in the subscore for rectal bleeding of $\geq 1$ point or an absolute subscore for rectal bleeding of $\leq 1 .^{21}$ Secondary endpoints were clinical remission rate (Mayo total score of $\leq 2$ and no individual score $>1$ ) and mucosal healing rate (Mayo total endoscopy score $\leq 1) .{ }^{21}$ Endoscopy was performed at screening and at Day 57 (or at early withdrawal).

Electronic patient diaries were used for Mayo subscore calculation. Patients recorded stool frequency and rectal bleeding on a daily basis. Recording was not permitted outside of the 24-h window. For prespecified primary and secondary endpoints, mean stool frequency and rectal bleeding scores were derived from values recorded in the 7 days prior to the study visit. If fewer than 3 days' values were available within 7 days (for either stool frequency or rectal bleeding), patients were defined as non-responders.

\section{Post hoc analyses}

In addition to the prespecified Mayo score analyses using the mean of 7 days of diary entries prior to the assessment date, post hoc analyses were also performed via the more conventional method of using the mean of 3 days of diary entries (diary scores were included if at least 1 day of diary entries were available) prior to assessment date for the stool frequency and rectal bleeding subscores.

Post hoc analyses were also performed for drug exposureresponse analyses. All patients in the intent-to-treat (ITT) population who had steady-state trough concentration $\left(\mathrm{C}_{\text {minss }}\right)$ values of BMS-93557 on Study Day 57 were stratified based on their 
$\mathrm{C}_{\text {minss }}$ values: low $(26.4-78.6 \mu \mathrm{g} / \mathrm{ml})$, mid $(79.2-105 \mu \mathrm{g} / \mathrm{ml})$ and top $(108-235 \mu \mathrm{g} / \mathrm{ml})$ tertiles. Clinical response, clinical remission and mucosal healing rates were calculated for each $\mathrm{C}_{\text {minss }}$ tertile, using the mean of 3 days of diary entries; 95\% CIs were provided using a Fisher's exact test. Logistic regression was used to study the drug exposure-response relationship between the $\mathrm{C}_{\text {minss }}$ and each of the efficacy measures. ORs associated with a doubling of the drug exposure were calculated along with their 95\% CIs; $\mathrm{p}$ values were also reported without multiplicity adjustment.

Histology analyses were also performed post hoc. Formalin-fixed biopsy samples were obtained at baseline and Day 57 (or at early withdrawal) from the section of the colon with the most endoscopically severe disease. Histology assessment was performed by one central pathologist (KG), blinded to study treatment; endoscopies were not recorded and there was no central endoscopy reader. Histology was scored using the Geboes Index, a six-grade classification system for inflammation specifying: 0 , structural change only; 1 , chronic inflammatory infiltrate; 2, lamina propria neutrophils; 3, neutrophils in epithelium; 4, crypt destruction; and 5, erosions or ulcers. ${ }^{23}$ Grading was based on the sample demonstrating the most histologically severe lesions. All patients who were biopsied during endoscopy at baseline and Day 57 and had two or more biopsy samples available for analysis at both time points are included in the histological analyses. Histological remission was assessed at Day 57, defined as a Geboes Index score of $<2.0$ (standard) $^{23}$ or $<1.0$ (stringent), ${ }^{20} 2425$ and is reported with 95\% CIs. Spidergram graphs were created for histological categories of BMS-936557-treated patients with $C_{\text {minss }} \geq 100 \mu \mathrm{g} / \mathrm{ml}$ and placebo patients at baseline and Day 57 in which each axis displays the six individual Geboes subscore components.

\section{Safety assessments}

The incidence and severity of adverse events (AEs) were monitored throughout the study and within 70 days after last study drug administration, including those which had worsened relative to pretreatment state and any treatment-related AE regardless of timing.

Related AEs were defined as those possibly, probably or definitely related to the study drug, with missing relationships presumed related. Peri-infusional events were defined as any AEs that could potentially constitute a reaction to infusion and occurred on the same day or the day after infusion. No prophylactic premedication was administered, unless indicated by previous infusion reaction experience in an individual patient. Vital sign monitoring, clinical laboratory tests, physical examinations, chest radiography and ECG were also performed.

Immunogenicity was assessed on Days 1, 29, 57 and 85 (42 days post last dose) using a validated electrochemiluminescent bridging immunoassay in human serum, using the Meso-Scale Discovery platform (Gaithersburg, Maryland, USA).

\section{Pharmacokinetics assessment}

Serum concentrations of BMS-936557 were assessed on Days 1, $8,15,29,43,57$ and 85 using a validated ELISA.

\section{Statistical analyses}

A sample size of 37 patients per group was necessary to make a statistically significant decision using a two-sided Fisher's exact test. This was based on expected response rates of $65.0 \%$ and $30.0 \%$ in the active and placebo groups, respectively. A total of 53 patients per group were required to account for an approximate $30.0 \%$ dropout rate over 8 weeks.

The efficacy measures, including the rates of clinical response, clinical remission and mucosal healing, were analysed using the ITT analysis population. Patients who discontinued from the study for any reason prior to reaching Day 57 were considered non-responders in the analysis of clinical response, clinical remission and mucosal healing. Differences in the rates of clinical response and clinical remission and mucosal healing between groups were assessed using Fisher's exact test, and 95\% CIs were determined for each treatment difference.

The pharmacokinetic analysis included patients who received at least one dose of BMS-936557 and had at least one concentration value; serum concentrations of BMS-936557 were summarised for Days 1, 8, 15, 29, 43, 57 and 85.

The safety analysis included all patients who had received at least one dose or partial dose of BMS-936557 or placebo, assessed through Day 113.
Figure 1 Patient disposition. *Two patients randomised to the placebo group were not treated.

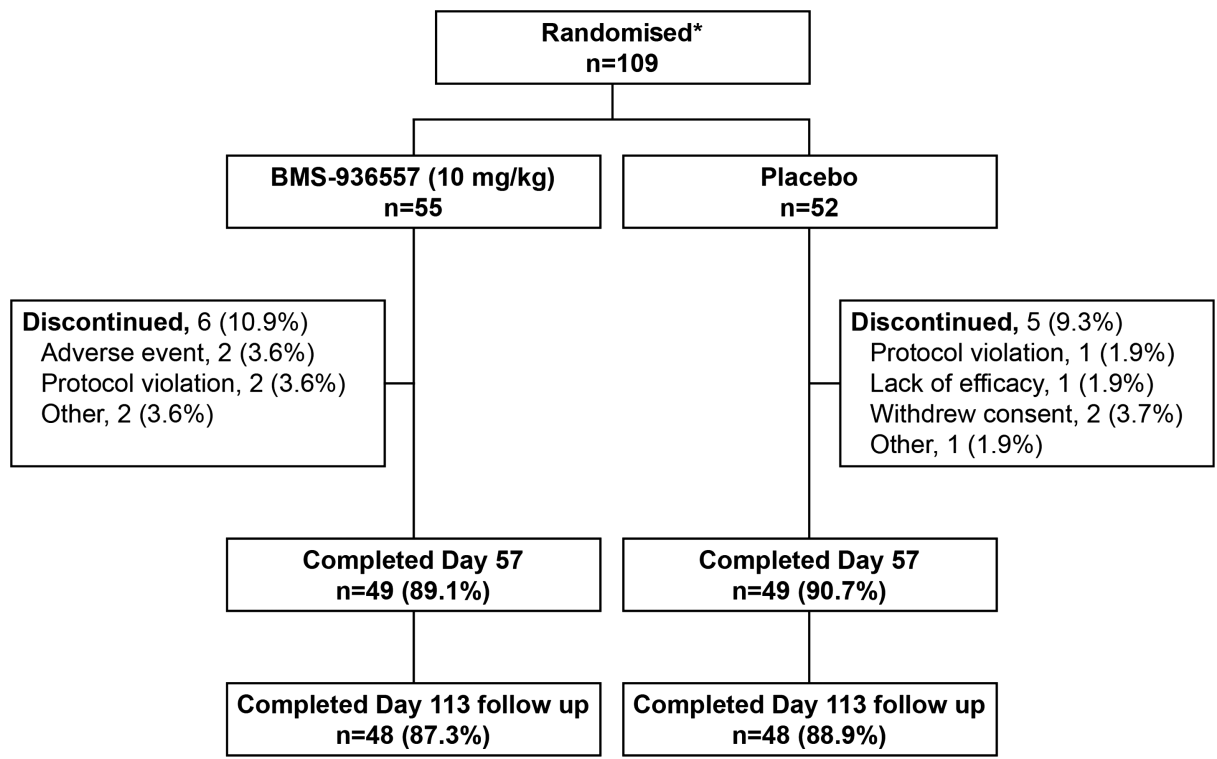


Table 1 Baseline demographics and disease characteristics

\begin{tabular}{|c|c|c|c|}
\hline & BMS-936557 n=55 & Placebo $n=54$ & Total $\mathrm{N}=109$ \\
\hline Mean age (SD) & $44.7(12.8)$ & $41.8(14.2)$ & $43.2(13.5)$ \\
\hline Mean weight, kg (SD) & $81.9(16.1)$ & $74.5(18.1)$ & $78.2(17.4)$ \\
\hline Male, n (\%) & $37(67.3)$ & $31(57.4)$ & $68(62.4)$ \\
\hline Race, \% Caucasian & $53(96.4)$ & $54(100)$ & $107(98.2)$ \\
\hline Country of enrolment, n (\%) & NA & NA & \\
\hline Czech Republic & & & $8(7.3)$ \\
\hline Hungary & & & $24(22.0)$ \\
\hline Latvia & & & $18(16.5)$ \\
\hline Romania & & & $11(10.1)$ \\
\hline Ukraine & & & $28(25.7)$ \\
\hline Russia & & & $9(8.3)$ \\
\hline North America & & & $11(10.1)$ \\
\hline Mean duration of UC, years (SD) & $6.7(7.8)$ & $5.5(4.4)$ & $6.1(6.4)$ \\
\hline \multicolumn{4}{|l|}{ Extent of disease, $\mathrm{n}(\%)$} \\
\hline Proctosigmoiditis & $15(27.3)$ & $13(24.1)$ & $28(25.7)$ \\
\hline Left-sided & $24(43.6)$ & $22(40.7)$ & $46(42.2)$ \\
\hline Extensive & $16(29.1)$ & $19(35.2)$ & $35(32.1)$ \\
\hline Mean Mayo score (SD) & $7.9(1.3)$ & $7.9(1.1)$ & NA \\
\hline Mean CRP, mg/dl (SD) & $1.3(2.0)$ & $1.2(1.4)$ & $1.3(1.7)$ \\
\hline \multicolumn{4}{|l|}{ Prior inadequate response/intolerance, $\mathrm{n}(\%)$} \\
\hline Immunosuppressants & $10(5.5)$ & $4(7.4)$ & $14(12.8)$ \\
\hline Anti-TNF & $3(5.5)$ & $3(5.6)$ & $6(5.5)$ \\
\hline Concomitant immunomodulator*, n (\%) & $6(10.9)$ & $10(18.5)$ & $16(14.7)$ \\
\hline Concomitant corticosteroids ${ }^{\dagger}, \mathrm{n}(\%)$ & $30(54.5)$ & $27(50.0)$ & $57(52.3)$ \\
\hline
\end{tabular}

\section{RESULTS}

\section{Patient population and baseline characteristics}

In total, 109 patients were randomised to BMS-936557 $(n=55)$ or placebo $(n=54)$. Patient disposition is shown in figure 1 . A total of $11(10.1 \%)$ patients discontinued prior to study completion at Day 57 (ITT analysis; six patients from the BMS-936557 group and five patients from the placebo group). Two (3.6\%) patients in the BMS-936557 group discontinued due to AEs; there were no discontinuations due to lack of efficacy in this group. No patients in the placebo group discontinued due to AEs; 1 (1.9\%) patient discontinued due to lack of efficacy (figure 1).

Baseline disease characteristics were generally similar between the two treatment groups (table 1). Although the mean disease duration was slightly longer in the BMS-936557 treatment group than in the placebo group, median disease duration was comparable in the two treatment groups (4.4 and 4.6 years for the BMS-936557 and placebo groups, respectively). During the study, the majority of patients in the BMS-936557 treatment group (85.5\%) and the placebo group (92.3\%) received all four planned doses of assigned treatment.

\section{Efficacy}

Prespecified analyses

The prespecified primary and secondary endpoints were not met in this study. At Day 57, the proportion of patients who achieved clinical response (primary endpoint) was numerically greater for the BMS-936557-treated group (52.7\%) versus the placebo-treated group (35.2\%) (figure 2) but this difference was not statistically significant (treatment difference $(95 \% \mathrm{CI}$ ) was $17.5 \%(-0.8$ to 35.9$))$. There were also no significant differences between the BMS-936557- and placebo-treated groups for clinical remission $(18.2 \%$ vs $16.7 \%)$ and mucosal healing $(41.8 \%$ vs $35.2 \%)$ (figure 2 ); treatment differences (95\% CI) were $1.5 \%(-12.7$ to 15.8$)$ and $6.6 \%(-11.6$ to 24.9), respectively.

\section{Post hoc analyses}

In the post hoc analysis (mean of 3 days of diary entries) of the primary and secondary endpoints, Day 57 clinical response rates were significantly higher in the BMS-936557 $(n=55)$ versus placebo $(n=54)$ group $(60.0$ vs $37.0 \%)$, with a treatment difference $(95 \% \mathrm{CI})$ of $23.0 \%(4.7,41.2$; Fisher's exact test: $\mathrm{p}=0.022)$. Remission and mucosal healing rates were numerically but not statistically higher in the BMS-936557 versus placebo group; $23.6 \%$ versus $18.5 \%$ (treatment difference $(95 \%$ CI) of $5.1 \%(-10.2$ to 20.4$)$, Fischer's exact test, $p=0.64)$ and $41.8 \%$ versus $35.2 \%$ (treatment difference $(95 \% \mathrm{CI}$ ) of $6.6 \%$ (-11.6 to 24.9$)$, Fischer's exact test, $p=0.556)$, respectively.

The geometric mean trough concentration $\left(\mathrm{C}_{\text {min }}\right)$ of BMS-936557 increased from $42.2 \mu \mathrm{g} / \mathrm{ml}$ on Day 15 to $91.3 \mu \mathrm{g} / \mathrm{ml}$ at steady-state on Day 57 . Higher trough concentration on Day $57\left(\mathrm{C}_{\text {minss }}\right)$ was associated with an increase in the clinical response rates (figure $3 \mathrm{~A}$ ). Significantly higher clinical response rates $(87.5 \%)$ were observed for the patients in the highest $\mathrm{C}_{\text {minss }}$ tertile subgroup (trough serum concentration at Day 57: $108-235 \mu \mathrm{g} / \mathrm{ml}$ ) compared with the placebo group $(37.0 \%) ; \mathrm{p}<0.001$, treatment difference $(95 \% \mathrm{CI})$ of $50.5 \%$ (29.8 to 71.2$)$. Logistic regression analysis also showed that an increase in $\mathrm{C}_{\text {minss }}$ resulted in an increase in clinical response (figure 3B), remission (figure 3C) and mucosal healing (data not shown). Population pharmacokinetic models have demonstrated 
Figure 2 Clinical response, remission and healing rates at Day 57 . Prespecified Mayo scoring method (intent-to-treat population). Mayo score re-derived after unblinding the data and by following conventional rules in the literature. Error bars represent 95\% Cls. A clinical response was defined as a decrease from baseline in the total Mayo score of at least 3 points and at least $30 \%$, with an accompanying decrease in the subscore for rectal bleeding of at least 1 point or an absolute subscore for rectal bleeding of 0 or 1 . Remission was defined as a total Mayo score of $\leq 2$ with no individual subscore exceeding 1 point and no blood in stools. Mucosal healing was defined as an absolute subscore for endoscopy of $\leq 1$.

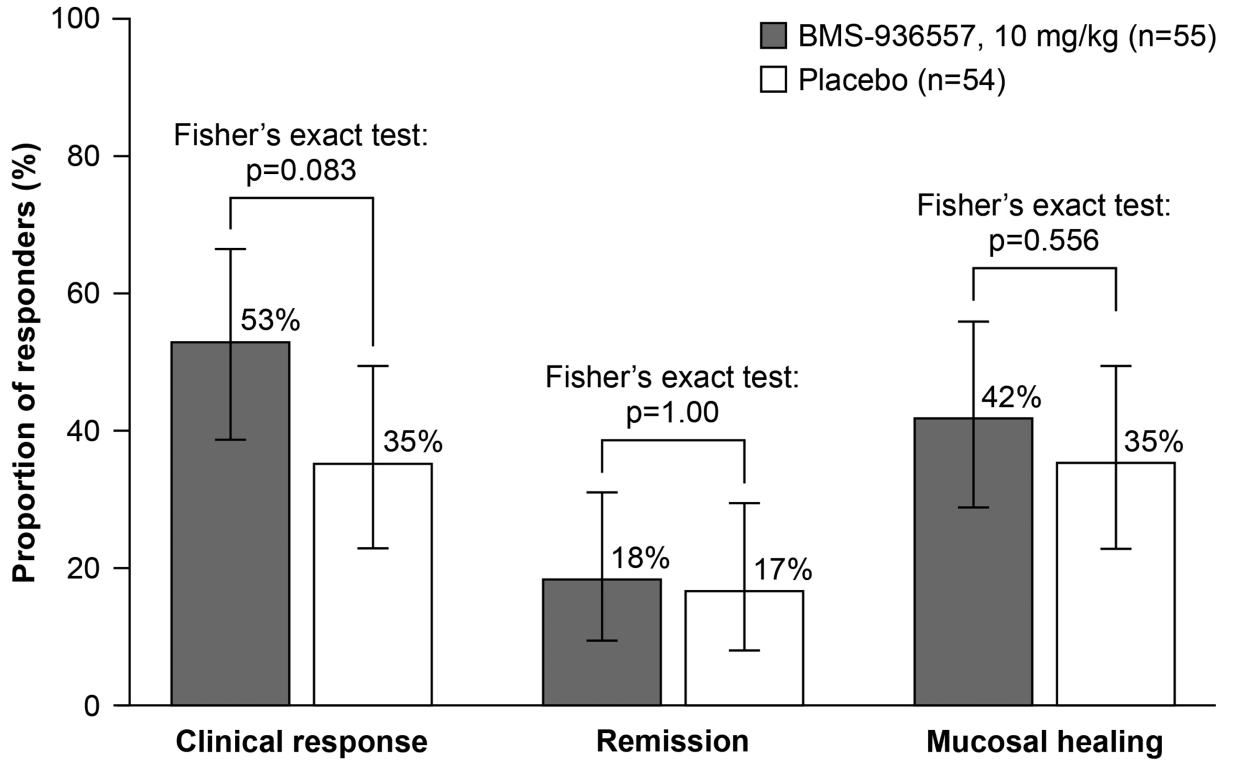

endoscopic remission (see online supplementary figure 1). Conversely, for placebo-treated patients, only those who achieve endoscopic remission appear to demonstrate histological improvement.

\section{Safety}

The serious adverse events (SAEs) in the BMS-936557 group were anaemia, localised infection, perforated appendicitis/ appendiceal abscess and diverticulitis in one patient each. In the placebo group, an SAE of $\beta$-hemolytic streptococcal infection was reported in one patient. All SAEs resolved without residual effects and were considered unlikely to be related, or unrelated, to study drug. There were no deaths reported during this study (table 2).

The BMS-936557-treated patient with the perforated appendicitis/appendiceal abscess discontinued treatment, as did another BMS-936557-treated patient with an event of vasculitis of moderate intensity, not considered an SAE. The patient with vasculitis presented with pain in the leg, accompanied by swelling of the left ankle following the first infusion of study medication. The event resolved within 2 weeks without residual effects.

For peri-infusional events, rash was reported in two patients in the BMS-936557 group; all other peri-infusional events (chest discomfort, feeling hot, pyrexia, tachycardia, headache, back pain, dyspnoea and flushing) were reported once. For placebo-treated patients, peri-infusional events included arthralgia, musculoskeletal pain and nausea. All peri-infusional events were mild-to-moderate in intensity, and none led to discontinuation. The majority of events were associated with the first infusion without recurrence at subsequent infusions.

The infections reported in the BMS-936557-treated group included cystitis, upper respiratory tract infection, appendiceal abscess/perforated appendicitis, diverticulitis, localised infection and oral herpes. No single type of infection occurred in more than two patients. In the placebo group, $\beta$-hemolytic streptococcal infection, nasopharyngitis and viral infection occurred in one patient each.

Analysis of the subgroup of patients with BMS-936557 trough concentrations $\geq 100 \mu \mathrm{g} / \mathrm{ml}$ showed similar safety results as described for the entire BMS-936557 safety population. The number of patients experiencing at least one $\mathrm{AE}$ in the $\mathrm{C}_{\text {minss }} \geq 100 \mu \mathrm{g} / \mathrm{ml}$ subgroup was comparable with that in the formed to further characterise the relationship between endoscopic healing and change in histology score, found that for patients treated with BMS-936557, there was a trend towards a decreased histology score regardless of whether patients achieve 

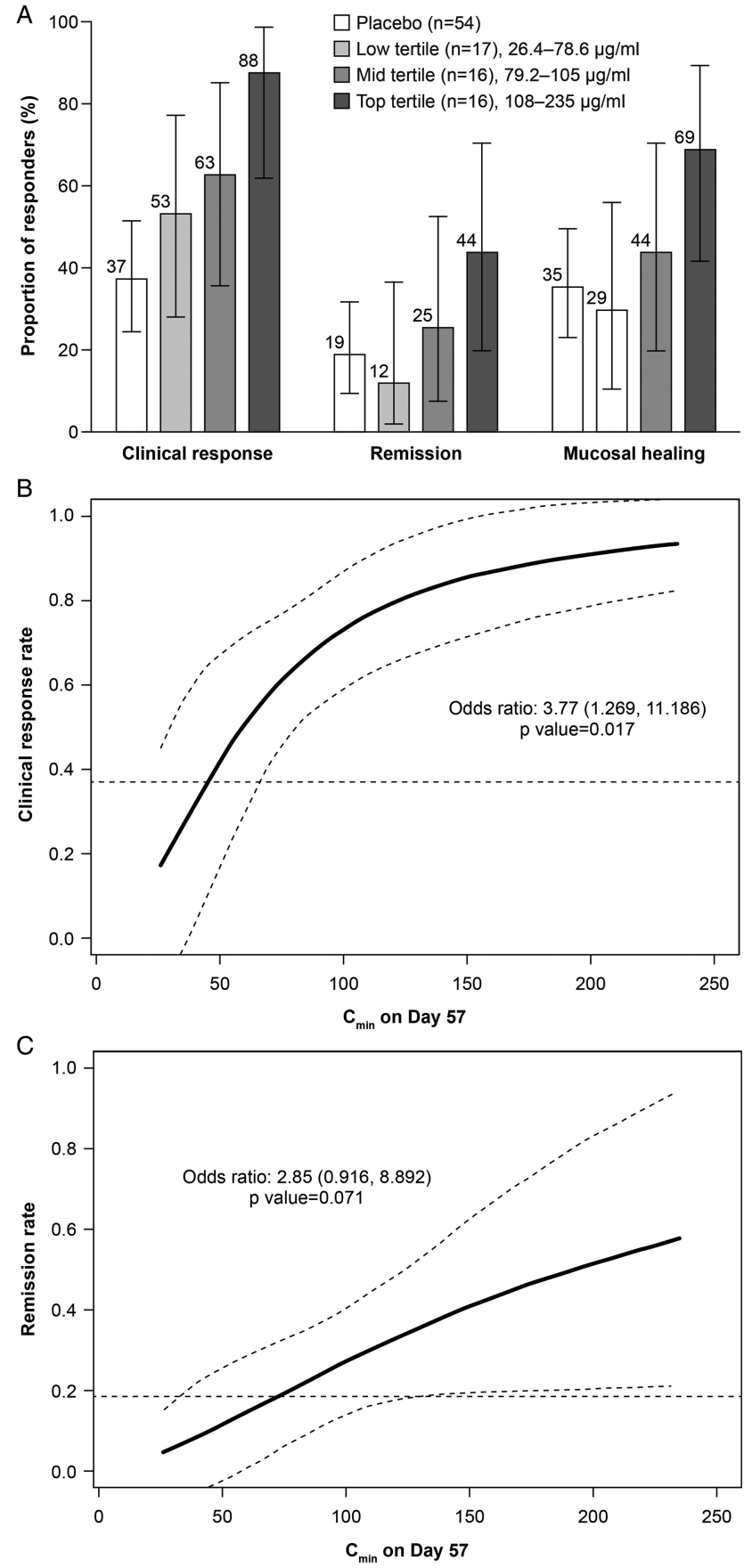

Figure 3 Clinical response by pharmacokinetic trough concentration at Day 57. (A) Post hoc analysis by trough concentration tertiles. Clinical response was defined as a decrease from baseline in the total Mayo score of at least 3 points and at least $30 \%$, with an accompanying decrease in the subscore for rectal bleeding of at least 1 point or an absolute subscore for rectal bleeding of 0 or 1 . Remission was defined as a total Mayo score of $\leq 2$ with no individual subscore exceeding 1 point and no blood in stools. Mucosal healing was defined as an absolute subscore for endoscopy of $\leq 1$. (B) Post hoc logistic regression analysis of clinical response by trough concentration. Patients from the placebo group were not included in this model fitting. Analysis demonstrated that by increasing BMS-936557 exposure by a factor of 2, the odds of achieving a clinical response increases by 3.77. Horizontal line denotes response rate of the placebo arm. (C) Post hoc logistic regression analysis of clinical remission by trough concentration. Patients from the placebo group were not included in this model fitting. Horizontal line denotes remission rate of the placebo arm. For all analyses, the Mayo score was re-derived after unblinding the data and by following conventional rules in the literature. placebo group. No AEs in the $C_{\text {minss }} \geq 100 \mu \mathrm{g} / \mathrm{ml}$ subgroup resulted in discontinuation from treatment. No immunogenicity was detected in any patient up to 42 days post last study dose.

\section{DISCUSSION}

Anti-IP-10 therapy with BMS-936557 demonstrated a modest effect at inducing a response in patients with moderate-to-severe UC in this proof-of-concept study. Although the prespecified primary and secondary endpoints were not met, post hoc subanalyses demonstrated a robust drug exposure-response relationship between the steady-state trough serum concentrations and efficacy: trough levels $\geq 100 \mu \mathrm{g} / \mathrm{ml}$ were found to result in significantly higher rates of clinical response, clinical remission and mucosal healing.

The original analysis of the Mayo score to assess the primary endpoint resulted in seven patients being imputed as nonresponders because they did not have the required number of days of diary records ( $\geq 3$ out of a possible 7) available for analysis. However, in the post hoc analysis (which analysed the data for patients with one or two records), clinical response rates were significantly higher in the BMS-936557 versus placebo group.

In addition to traditional endoscopy, histological analysis of biopsies can facilitate an additional assessment of disease activity, particularly in endoscopically non-inflamed mucosa. ${ }^{23}$ In this study, significant histological improvements were observed in patients with $C_{\text {minss }} \geq 100 \mu \mathrm{g} / \mathrm{ml}$, even in some patients who did not demonstrate significant clinical improvement. Furthermore, significantly more patients receiving BMS-936557 (with $\mathrm{C}_{\text {minss }} \geq 100 \mu \mathrm{g} / \mathrm{ml}$ ), versus placebo, achieved a Geboes score $<2$ at Day 57 (73\% vs 41\%, respectively), with improvements observed across all histological categories. Results were similar using a lower Geboes score cut-off of $<1$ (structural change only), with $47 \%$ versus $18 \%$ of BMS-936557 (with $\mathrm{C}_{\text {minss }} \geq 100 \mu \mathrm{g} / \mathrm{ml}$ ) patients versus placebo patients achieving remission. In the Active Ulcerative Colitis 1 (ACT 1) study of infliximab, $35 \%$ of infliximab-treated patients achieved this outcome compared with $24 \%$ of placebo-treated patients following 8 weeks of treatment. ${ }^{24}$ Although not yet validated, the Geboes index has demonstrated good reproducibility and modest agreement with the endoscopic grading system, and represents a histological activity system of great potential value in both the clinical trial and clinical practice settings. ${ }^{23}$

Data from histological analyses complement the clinical data, and are of particular interest, as they offer insight into the mechanism of action of BMS-936557. IP-10 is directly and indirectly involved in the activation and/or recruitment of pro-inflammatory cells responsible for inflammation and tissue destruction, ${ }^{5}$ and treatment with BMS-936557 resulted in a marked reduction in overall inflammatory infiltrate, and specifically in the level of acute inflammatory infiltrates (particularly in patients with BMS-936557 exposure levels $\geq 100 \mu \mathrm{g} / \mathrm{ml}$ ). Furthermore, IP-10 has also been shown to play a role in the modulation of proliferation and migration of epithelial cells, ${ }^{9}$ and treatment with BMS-936557 (above the target exposure) was associated with a marked decrease in erosion, ulceration and crypt destruction. These observations suggest that BMS-936557 inhibits IP-10-induced epithelial cell dysfunctions, either directly or indirectly, resulting in increased barrier integrity. The impact of BMS-936557 on histological remission rate suggests that BMS-936557 induces histological healing, which can only be achieved by targeting histological inflammation and associated tissue damage. ${ }^{1}$ 

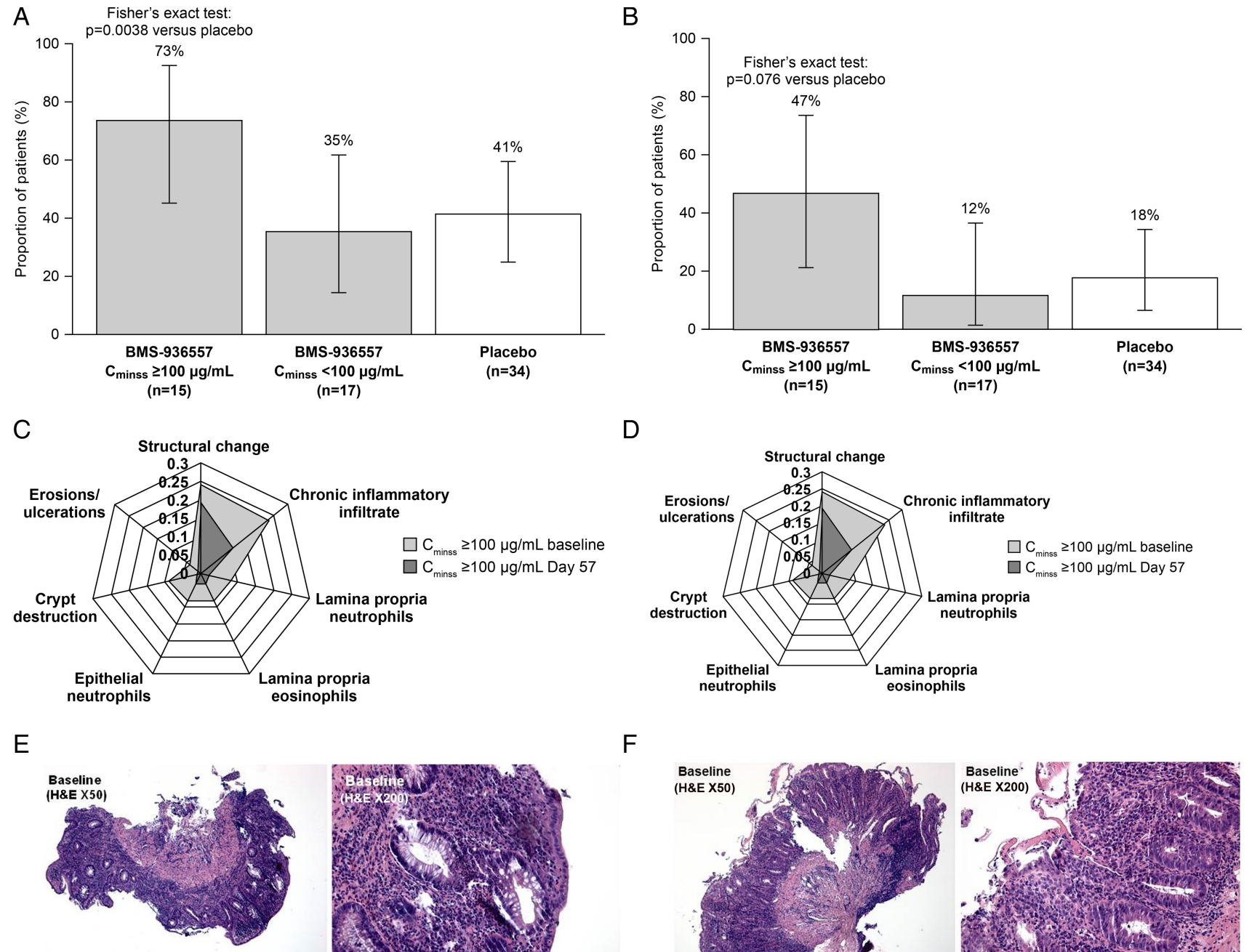

$\mathrm{F}$
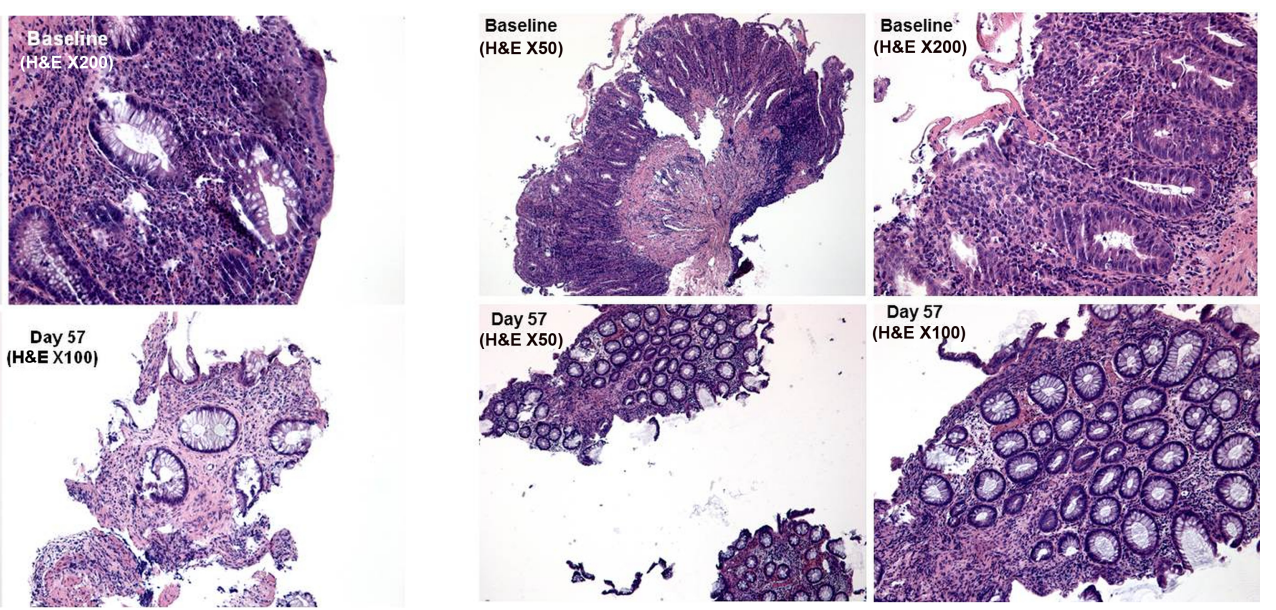

Figure 4 Histological analyses at Day 57. (A) Proportion of patients in histological remission (Geboes Index score $<2$ ) at Day 57. (B) Proportion of patients in histological remission (Geboes Index score $<1$ ) at Day 57. (C) Improvement across histological categories for BMS-936557-treated patients with $C_{\text {minss }} \geq 100 \mu \mathrm{g} / \mathrm{ml}(n=15) ; C_{\text {minss }}=$ steady-state trough serum concentration; Error bars represent $95 \% \mathrm{Cl}$. (D) Improvement across histological categories for placebo-treated patients $(n=32)$. (E) Histological improvement in the presence of clinical improvement in a patient who achieved BMS-936557 $C_{\text {minss }} \geq 100 \mu \mathrm{g} / \mathrm{ml}$. Baseline Mayo score was 8 and endoscopy subscore was 3; Day 57 Mayo score was 2 and endoscopy subscore was 1. Baseline Geboes Index score was 5, and H\&E staining shows mucosa of the colon with moderate architectural abnormalities: crypts are shortened, not reaching the muscularis mucosae; variable intercryptal distance. Epithelial cells are not well differentiated. The cellular infiltrate in the lamina propria is severely increased with basal accumulation; composition is mixed, with mild cryptitis. Day 57 Geboes Index score was 0 , and $\mathrm{H} \& \mathrm{E}$ staining shows mucosa of the colon with moderate architectural abnormalities; variable intercryptal distance. Epithelial cells are well differentiated (surface loss is due to artefact) and cellular infiltrate in the lamina propria is within normal limits. (F) Histological improvement in the absence of clinical improvement in a patient who achieved BMS-936557 $C_{\text {minss }} \geq 100 \mu \mathrm{g} / \mathrm{ml}$. Baseline Mayo score was 8 and endoscopy subscore was 2; Day 57 Mayo score was 7 and endoscopy subscore was 2. Baseline Geboes Index score was 5 . H\&E staining shows mucosa of the colon with moderate architectural abnormalities: crypts are shortened, not reaching the muscularis mucosae; variable intercryptal distance. Epithelial cells are less well differentiated - surface epithelial cells are flattened and partially lost: at the edge of the loss, the cells are flattened indicating restitution (part of the healing process). The cellular infiltrate in the lamina propria is severely increased with basal accumulation; composition is mixed, with mild cryptitis. At Day 57, Geboes Index score was 0. H\&E staining shows colon with normal architecture: surface is smooth and crypts run parallel. Internal diameter of crypts is constant and distance between crypts is normal and constant. Mucin secretion is well preserved and the lamina propria infiltrate is borderline normal in distribution and intensity. Epithelial cells are well differentiated. In the $\times 100$ magnification, there is some lifting of surface epithelial cells, which can be explained as an artefact as the cells are well differentiated. All analyses were performed post hoc. Access the article online to view this figure in colour. 
Table 2 Summary of AEs

\begin{tabular}{lcc}
\hline $\mathbf{n}(\%)$ & BMS-936557 $\mathbf{n = 5 5}$ & Placebo $\mathbf{n = 5 2}$ \\
\hline AEs & $22(40.0)$ & $17(32.7)$ \\
Related AEs & $11(20.0)$ & $7(13.5)$ \\
AEs resulting in discontinuation & $2(3.6)$ & 0 \\
SAEs & $4(7.3)$ & $1(1.9)$ \\
Serious infections & $3(5.5)$ & $1(1.9)$ \\
Deaths & 0 & 0 \\
Infections and infestations & $7(12.7)$ & $3(5.8)$ \\
Peri-infusional AEs* & $6(10.9)$ & $2(3.8)$ \\
\hline
\end{tabular}

*Infusion reaction within $24 \mathrm{~h}$ of infusion. All Grade 1-2, all recovered and none led to early termination; clinical laboratory data, vital signs, physical examinations, ECG or chest radiographs assessed during the study did not highlight any safety concerns; safety was assessed through Day 113.

AE, adverse event; SAE serious adverse event.

BMS-936557 was generally safe and well tolerated. There appeared to be a numerically higher frequency of infections, including serious infections in the BMS-936557 group compared with placebo. In a previous phase II study of BMS-936557 in patients with rheumatoid arthritis, the frequency of infections was similar in BMS-936557- and placebo-treated patients. ${ }^{25}$ While all peri-infusion reactions were mild to moderate and none led to early discontinuation, the frequency of events appeared to be higher in the BMS-936557 group compared with placebo. No immunogenicity was detected in any patient (up to 42 days post last study dose).

As highlighted above, the drug exposure-response results demonstrated that higher trough serum levels at Day 57 were associated with higher rates of clinical response, clinical remission, histological remission and mucosal healing, whereas the safety profile observed in patients with higher trough levels was comparable with the overall study population. Collectively, these findings suggest that it may be beneficial to investigate doses of BMS-936557 greater than $10 \mathrm{mg} / \mathrm{kg}$ in future trials.

The data presented here should be interpreted within the context of the trial's limitations. The primary endpoint was not met in the overall population; although robust efficacy was demonstrated in the subgroup of patients with higher BMS-936557 exposure, the trial was not originally powered to detect statistical differences between active and control treatments in groups of this size. Subgroup and as-observed histology assessments were performed post hoc and should be interpreted with caution owing to the known limitations of such analyses. In addition, histology assessments were carried out for all patients with sufficient available samples, but biopsies were not performed on every patient. Therefore, the possibility of sample selection bias must be considered. It should be noted that the baseline disease severity of this study population was milder compared with other recent studies in moderately-to-severely active UC; ${ }^{26-28}$ patients with a baseline Mayo score of greater than 10 were excluded and only $14 \%$ of patients were refractory to immunosuppressants and $6 \%$ to anti-TNF agents. The placebo response/remission rate observed in this study is comparable with other studies with biological therapies in moderately-to-severely active UC. ${ }^{28}{ }^{29}$ Furthermore, evaluating study results for global studies may be complicated by variations in results between patients from different geographic locations, in particular Eastern Europe and North America. However, of the 109 patients randomised, only 11 were from North America, specifically the USA, and given this small sample size, post hoc analyses by geographic region were not conducted.
In conclusion, the anti-IP-10 antibody, BMS-936557, is a potentially effective therapy for moderately-to-severely active UC. Higher drug exposure was associated with increasing clinical response and histological improvement. Further doseresponse studies are warranted.

Acknowledgements The authors would like to thank the investigators at the study sites: F Anderson, L Augustin, O Babak, L Barany, J Baz, A Buchman, P Cardarelli, Z Csiki, D Daniela, O Datsenko, D Dejica, C Diaz Stone, A Dorofeyev, L Douda, B Gasztonyi, L Gheorghe, A Goldis, M Gould, M Gurzo, Z Gurzo, R Hardi, A Hemaidan, I Horny, W Hunter Eubanks, B Hunyady, N Kakurina, J Kersak, OA Khrustalev, I Klyarits'ka, P Kohout, M Konecny, J Kotrlik, D Lisova, Y Lozynskyi, G Lysenko, M Lukas, L Mayer, T Mikhailova, O Minushkin, Z Nemecek, TJ Patrick, H Pluta, J Pokrotnieks, I Polianskyi, E Popescu, VM Radu, Y Shvarts, A Svintsitskyy, T Szaloki, M Tichy, I Tolmanis, S Valean, Y Zakharash and F Zsigmond.

Contributors All authors have been involved in analysis and interpretation of the data. All authors have critically reviewed the manuscript draft for intellectual content and approved the final version of the manuscript for publication. The corresponding author had full access to the data and takes full responsibility for the veracity of the data and statistical analysis.

Funding This study was funded by Bristol-Myers Squibb, Princeton, New Jersey, USA. Editorial assistance was provided by Róisín O'Connor, Medicus International, and funded by Bristol-Myers Squibb.

Competing interests LM has received consulting fees from Abbott, NovoNordisk, Bristol-Myers Squibb, Lexicon, Amgen and Neovacs. WJS has received consulting fees from Abbott, ActoGeniX NV, AGI Therapeutics Inc, Alba Therapeutics Corp, Albireo, Alfa Wasserman, Amgen, AM-Pharma BV, Anaphore, Astellas, Athersys Inc, Atlantic Healthcare, Aptalis, BioBalance Corp, Boehringer-Ingelheim, Bristol-Myers Squibb, Celgene, Celek Pharmaceuticals, Cellerix SL, Cerimon Pharmaceuticals, ChemoCentryx, CoMentis, Cosmo Technologies, Coronado Biosciences, Cytokine Pharmasciences, Eagle Pharmaceuticals, EnGene Inc, Eli Lilly, Enteromedics, Exagen Diagnostics Inc, Ferring Pharmaceuticals, Flexio Therapeutics Inc, Funxional Therapeutics, Genzyme Corp, Gilead Sciences, Given Imaging, GSK, Human Genome Sciences, Ironwood Pharmaceuticals, KaloBios Pharmaceuticals, Lexicon Pharmaceuticals, Lycera Corp, Meda Pharmaceuticals, Merck Research Laboratories, Merck Serono, Millennium Pharmaceuticals, Nisshin Kyorin Pharmaceuticals, Novo Nordisk, NPS Pharmaceuticals, Optimer Pharmaceuticals, Orexigen Therapeutics Inc, PDL Biopharma, Pfizer, Procter and Gamble, Prometheus Laboratories, ProtAb, Purgenesis Technologies Inc, Relypsa Inc, Roche, Salient Pharmaceuticals, Salix Pharmaceuticals, Santarus, Schering Plough, Shire Pharmaceuticals, Sigmoid Pharma, Sirtris Pharmaceuticals, SLA Pharma UK, Targacept, Teva Pharmaceuticals, Therakos, Tilliotts Pharma AG, TxCell SA, UCB Pharma, Viamet Pharmaceuticals, Vascular Biogenics, Warner Chilcott UK and Wyeth; research grants from Abbott, Bristol-Myers Squibb, Genentech, GSK, Janssen, Millennium Pharmaceuticals, Novartis, Pfizer, Procter and Gamble, Shire Pharmaceuticals and UCB Pharma; payments for lectures/speaker bureaux from Abbott, Bristol-Myers Squibb and Janssen; and holds stock/stock options in Enteromedics. YS, KG and RH have nothing to disclose. MY was an employee of Medarex during the design and conduct of the study and owned Medarex stock. XLT, LAX, LS-C and AYL are all employees of Bristol-Myers Squibb and hold stock options. SG and RA were employees of Bristol-Myers Squibb at the time of data analyses; RA held stock options in Bristol-Myers Squibb at the time of data analysis. Potential conflicts of interest were disclosed to study participants during this trial.

Ethics approval Ethics approval was provided by the local ethics committee.

Provenance and peer review Not commissioned; externally peer reviewed.

Open Access This is an Open Access article distributed in accordance with the Creative Commons Attribution Non Commercial (CC BY-NC 3.0) license, which permits others to distribute, remix, adapt, build upon this work non-commercially, and license their derivative works on different terms, provided the original work is properly cited and the use is non-commercial. See: http://creativecommons.org/licenses/by-nc/3.0/

\section{REFERENCES}

1 Ordas I, Eckmann L, Talimini M, et al. Ulcerative colitis. Lancet 2012;380:1606-19.

2 Kornbluth A, Sachar DB. Ulcerative colitis practice guidelines in adults: American College Of Gastroenterology, Practice Parameters Committee. Am J Gastroenterol 2010;105:501-23; quiz 24.

3 Travis SP, Stange EF, Lemann M, et al. European evidence-based Consensus on the management of ulcerative colitis: current management. J Crohns Colitis 2008;2:24-62.

4 Lichtenstein GR, Abreu MT, Cohen R, et al. American Gastroenterological Association Institute technical review on corticosteroids, immunomodulators, and infliximab in inflammatory bowel disease. Gastroenterology 2006;130:940-87. 
5 Kuhne MPB, Wallace $S$, Chen S, et al. MDX-1100, a fully human anti-CXCL 10 (IP-10) antibody, is a high affinity, neutralizing antibody that has intered phase I clinical trials for the treatment of Ulcerative Colitis (UC). J Immunol 2007;178:20.

6 Kabashima H, Yoneda M, Nagata K, et al. The presence of chemokine (MCP-1, MIP-1(alpha), MIP-1(beta), IP-10, RANTES)-positive cells and chemokine receptor (CCR5, CXCR3)-positive cells in inflamed human gingival tissues. Cytokine 2002:20:70-7.

7 Molesworth-Kenyon S, Mates A, Yin R, et al. CXCR3, IP-10, and Mig are required for CD4+ T cell recruitment during the DTH response to HSV-1 yet are independent of the mechanism for viral clearance. Virology 2005;333:1-9.

8 Romagnani P, Maggi L, Mazzinghi B, et al. CXCR3-mediated opposite effects of CXCL10 and CXCL4 on TH1 or TH2 cytokine production. J Allergy Clin Immunol 2005;116:1372-9.

9 Suzuki K, Kawauchi Y, Palaniyandi SS, et al. Blockade of interferon-gammainducible protein-10 attenuates chronic experimental colitis by blocking cellular trafficking and protecting intestinal epithelial cells. Pathol Int 2007;57: 413-20.

10 Soejima K, Rollins BJ. A functional IFN-gamma-inducible protein-10/CXCL10-specific receptor expressed by epithelial and endothelial cells that is neither CXCR3 nor glycosaminoglycan. J Immunol 2001;167:6576-82.

11 Schulthess FT, Paroni F, Sauter NS, et al. CXCL10 impairs beta cell function and viability in diabetes through TLR4 signaling. Cell Metab 2009;9:125-39.

12 Luster AD, Greenberg SM, Leder P. The IP-10 chemokine binds to a specific cell surface heparan sulfate site shared with platelet factor 4 and inhibits endothelial cell proliferation. J Exp Med 1995;182:219-31.

13 Uguccioni M, Gionchetti P, Robbiani DF, et al. Increased expression of IP-10, IL-8, MCP-1, and MCP-3 in ulcerative colitis. Am J Pathol 1999;155:331-6.

14 Witte A, Kuhne MR, Preston BT, et al. W1170 CXCL10 expression and biological activities in inflammatory bowel disease. Gastroenterology 2008;134:A-648.

15 Sasaki S, Yoneyama $\mathrm{H}$, Suzuki $\mathrm{K}$, et al. Blockade of CXCL10 protects mice from acute colitis and enhances crypt cell survival. Eur I Immunol 2002;32:3197-205

16 Singh UP, Singh S, Taub DD, et al. Inhibition of IFN-gamma-inducible protein-10 abrogates colitis in IL-10-/- mice. J Immunol 2003;171:1401-6.

17 Hyun JG, Lee G, Brown JB, et al. Anti-interferon-inducible chemokine, CXCL10, reduces colitis by impairing $T$ helper-1 induction and recruitment in mice. Inflamm Bowel Dis 2005:11:799-805.
18 Mayer L, Sandborn WJ, Stepanov Y, et al. A randomized placebo-controlled trial of MDX-1100, an anti-IP-10 antibody, for moderately-to-severely active ulcerative colitis. Oral presentation at DDW (Abstract 711a) 2010.

19 Hardi R, Mayer L, Targan SR, et al. A phase 1 open-label, single-dose, dose-escalation study of MDX-1100, a high-affinity, neutralizing, fully human lgg1 (kappa) anti-CXCL10 (Ip10) monoclonal antibody, in ulcerative colitis. Gastroenterology 2008;134:A-99-100.

20 Yellin M, Paliienko I, Balanescu A, et al. A phase II, randomized, double-blind, placebo-controlled study evaluating the efficacy and safety of MDX-1100, a fully human anti-CXCL10 monoclonal antibody, in combination with methotrexate in patients with rheumatoid arthritis. Arthritis Rheum 2011;64:1730-9.

21 D'Haens G, Sandborn WJ, Feagan BG, et al. A review of activity indices and efficacy end points for clinical trials of medical therapy in adults with ulcerative colitis. Gastroenterology 2007:132:763-86.

22 Schroeder KW, Tremaine WJ, Ilstrup DM. Coated oral 5-aminosalicylic acid therapy for mildly to moderately active ulcerative colitis. A randomized study. N Engl J Med 1987;317:1625-9.

23 Geboes K, Riddell R, Ost A, et al. A reproducible grading scale for histological assessment of inflammation in ulcerative colitis. Gut 2000;47:404-9.

24 Geboes K, Olson A, Marano C. Infliximab results in reduction of inflammation and inflammatory markers in the mucosa of ulcerative colitis patients: the ACT 1 trial (Abstract). Am J Gastroenterol 2005;100:S292-3.

25 Yellin M, Paliienko I, Balanescu A, et al. A phase 2, multi-dose, double-blind, placebo-controlled, randomized, multicenter study of MDX-1100 (anti-CXCL10 Human Monoclonal Antibody) in combination with methotrexate in patients with active rheumatoid arthritis. Arthritis Rheum 2009;60:414.

26 Afif W, Leighton JA, Hanauer SB, et al. Open-label study of adalimumab in patients with ulcerative colitis including those with prior loss of response or intolerance to infliximab. Inflamm Bowel Dis 2009;15:1302-7.

27 Baumgart DC, Targan SR, Dignass AU, et al. Prospective randomized open-labe multicenter phase I/II dose escalation trial of visilizumab (HuM291) in severe steroid-refractory ulcerative colitis. Inflamm Bowel Dis 2010;16:620-9.

28 Rutgeerts P, Sandborn WJ, Feagan BG, et al. Infliximab for induction and maintenance therapy for ulcerative colitis. N Engl J Med 2005;353:2462-76.

29 Sandborn W, Colombel J-F, Hanauer S, et al. A randomized placebo-controlled trial of abatacept for moderately-to-severely active ulcerative colitis. DDW abstract 2010. 